\title{
Design of crossed-mirror array to form floating 3D LED signs
}

Hirotsugu Yamamoto, Hiroki Bando, Ryousuke Kujime, Shiro Suyama

Hirotsugu Yamamoto, Hiroki Bando, Ryousuke Kujime, Shiro Suyama, "Design of crossed-mirror array to form floating 3D LED signs," Proc. SPIE 8288, Stereoscopic Displays and Applications XXIII, 828820 (28 February 2012); doi: 10.1117/12.909879

SPIE Event: IS\&T/SPIE Electronic Imaging, 2012, Burlingame, California, United States 


\title{
Design of crossed-mirror array to form floating 3D LED signs
}

\author{
Hirotsugu Yamamoto*a, ${ }^{\text {, }}$ Hiroki Bando ${ }^{\text {a }}$, Ryousuke Kujime ${ }^{\mathrm{a}}$, Shiro Suyama ${ }^{\mathrm{a}}$ \\ ${ }^{a}$ Department of Optical Science and Technology, Faculty of Engineering, University of Tokushima, \\ Minamijosanjima 2-1, Tokushima 770-8506, Japan; \\ ${ }^{\mathrm{b} J S T, ~ C R E S T, ~ M i n a m i j o s a n j i m a ~ 2-1, ~ T o k u s h i m a ~ 770-8506, ~ J a p a n . ~}$
}

\begin{abstract}
3D representation of digital signage improves its significance and rapid notification of important points. Our goal is to realize floating 3D LED signs. The problem is there is no sufficient device to form floating 3D images from LEDs. LED lamp size is around $1 \mathrm{~cm}$ including wiring and substrates. Such large pitch increases display size and sometimes spoils image quality. The purpose of this paper is to develop optical device to meet the three requirements and to demonstrate floating 3D arrays of LEDs. We analytically investigate image formation by a crossed mirror structure with aerial aperture, called CMA (crossed-mirror array). CMA contains dihedral corner reflectors at each aperture. After double reflection, light rays emitted from an LED will converge into the corresponding image point. We have fabricated CMA for 3D array of LEDs. One CMA unit contains 20 x 20 apertures that are located diagonally. Floating image of LEDs was formed in wide range of incident angle. The image size of focused beam agreed to the apparent aperture size. When LEDs were located three-dimensionally (LEDs in three depths), the focused distances were the same as the distance between the real LED and the CMA.
\end{abstract}

Keywords: Crossed mirror array, LED, floating image, volumetric display, dihedral corner reflectors

\section{INTRODUCTION}

Crossed mirrors, sometimes called Lobster's eye optics, are widely used for X-ray optics [1-4]. As they are crossed mirrors have a wide field of angle, a deep depth of focus, and no chromatic aberration. A roof mirror lens array also enables unit magnification imaging [5]. Recently, Maekawa developed a special optical component to form threedimensional (3D) image on the mirrored symmetry position [6]. The optical component is composed of dihedral corner reflectors, which are manufactured by high precision techniques to provide a sharp image. Volumetric 3D display has been realized by use of his optical element [7]. Although we have realized several types of stereoscopic LED display by use of a large parallax barrier [8-12], floating LED signage has not realized yet. There was no optical elements that are suitable to form a floating image of a large LED panel.

In this paper, we propose a three-dimensionally floating display of LED signage. Floating display provide a higher sense of reality than conventional stereoscopic 3D display with glasses because three-dimensionally floating image is visible with the naked eyes. We can place the sign message at the exactly three-dimensional position. Furthermore, one of the significant advantages in using floating display for digital signage is its mechanically transparent feature, that is, there is no physical contact on the floating image. Fig. 1 illustrates an application of floating LED signage for traffic sign. Floating "STOP" warning appears just in front of the driver's seat. Since the sign is an aerial image, the sign cannot be crashed even if the car goes through it.

These alarm signs are visible over a wide viewing angle. Then, the optical elements are required wide (at least 45 deg.) field of angle. For such installations as road traffic and other public facilities, the optical elements are required to be secure against crashing under severe earthquakes. In order to form floating image of an LED array of high brightness, wide point spread function is suitable because there are black areas between LED lamps. Furthermore, the required size of the optical elements is larger than our body in future. Then, scalability of composition is needed.

The purpose is to realize a new optical element to form floating image of an LED panel. We investigate crossed mirrors because crossed-mirror structure with aerial aperture enables wide field of angle. We call this type of optical device as CMA (crossed-mirror array), hereafter. Image formation principle are analyzed and realized experimentally.

*yamamoto@opt.tokushima-u.ac.jp; phone 8188 656-9426; fax 8188 656-9435; www.opt.tokushima-u.ac.jp 


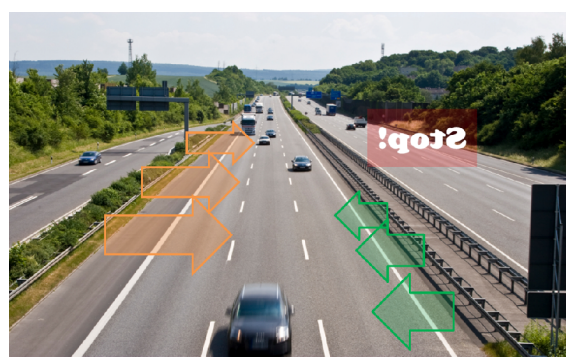

Figure 1. Application of floating 3D LED signage for traffic signs. Arrows and stop signs will be represented right in front of moving cars at the right distances. Cars go through the signs because the signs are physically transparent.

In order to provide wide point spread function, aperture ratio is 0.8 horizontally and vertically. Formation of floating image with a certain spread has been investigated with prototype elements. Furthermore, floating display of an LED sign has been demonstrated.

In Section 2, design principle of CMA for LEDs is described. In Section 3, we report experimental results on image forming and demonstrate floating display of an LED array.

\section{DESIGN OF CROSSED-MIRROR ARRAY FOR LED PANEL}

\subsection{Design philosophy of CMA for LED panel}

Reflective optical elements have been commonly used for imaging in the field of X-ray optics. Fig. 2 shows how dihedral corner reflectors act as an image-forming optical element. After double reflection, the light is always reflected to the reversed direction of the incident light in the plane parallel to the optical element and toward the other side of the element in the plane perpendicular to element. Then, light rays emitted from an object point will converge into the corresponding image point. Each aperture in CMA performs as dihedral corner reflectors. Compared to imaging using lens to form floating 3D image, benefits in using reflection based optical elements include low distortion image, no chromatic aberration, wide field of view, and deep depth of focus. However, there is a shift of the exit light ray and the shift depends on the reflection position of the dihedral corner reflector. Thus, narrow aperture is required in order to perform fine imaging in conventional design.

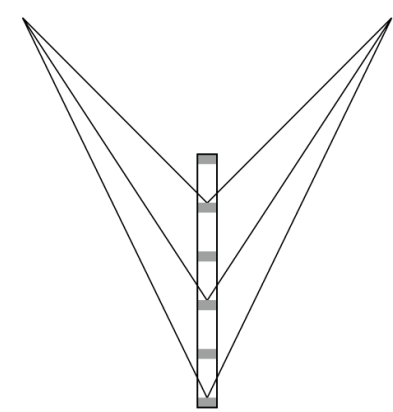

FRONT VIEW

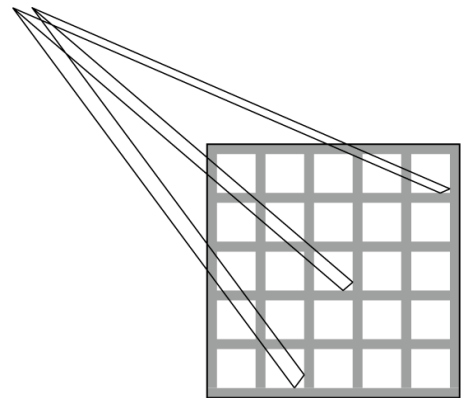

SIDE VIEW

Figure 2. Image formation by use of a reflective optical element that is composed of dihedral corner reflectors, which is sometime called RMGA (roof mirror grid array). After double reflections, incident lights from the same light source converge to the image point, which is located at the planer symmetry position of the light source regarding to the reflective optical element. The size of aperture and the thickness of the reflective optical element influence the size of the point spread function.

The key point of designing CMA for LED is to provide a appropriately wide point spread function. Because LED panels for large digital signage are consisted of sparse LED lamps as shown in Fig. 3 (a). Defocusing of an LED panel gives smooth outline of the letter as shown in Fig. 3 (b). Fig. 4 shows difference in the fundamental design philosophy. Conventional dihedral corner reflectors are composed of narrow apertures to maintain a sub-millimeter point spread function to form clear image of flat panel displays. This is because incident rays at different position in the same aperture are reflected into a slightly different position, although incident rays into many apertures are reflected and converged toward the imaging position that is the plane-symmetrical position of the light source. Geometrically, the point spread 
function is considered to be related with the aperture size. Designing a CMA with a wide aperture and thick elements is a challenging issue.

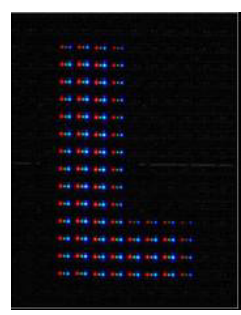

(a)

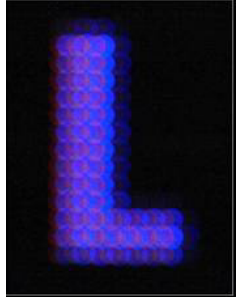

(b)

Figure 3. Photographs of LED signs taken by (a) focusing the LED panel and (b) defocusing the LED panel. In this case lamp type LED are located at $20 \mathrm{~mm}$ pitch. Black regions between LED lamps are significant when picture was taken by focusing the LED panel. By defocusing the LED panel, the black regions are removed by filling LED lights and smooth shape of $\mathrm{L}$ is easily perceived.

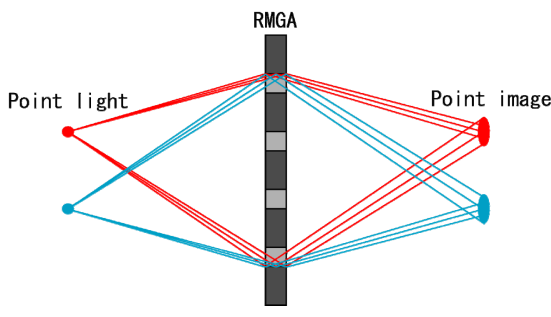

(a)

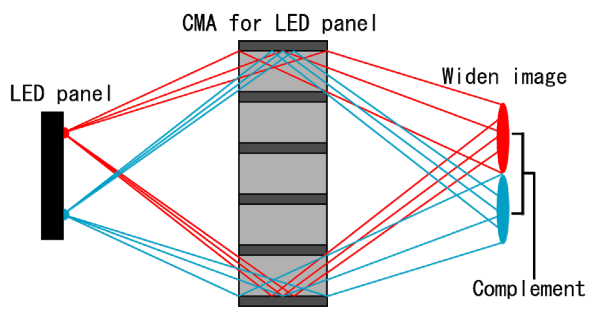

(b)

Figure 4. Schematic diagram showing difference of design principle of reflective optical elements. (a) Conventionally, narrow apertures and thin components are fabricated in order to form fine image. (b) In order to fulfill the gaps between LED lamps, wide apertures and thick components provide wide point spread function.

\subsection{Point spread function in image forming by use of CMA}

In this section, image formation principle by use of CMA is analyzed in Cartesian coordinates, as shown in Fig. 5. Let a CMA is placed on $x y$-plane. The origin of the coordinates is located at one of the corners of CMA. The CMA is composed of mirrors with plate thickness $T$, aperture width $W$, and mirror height $H$.

A light ray emitted from the point light source position enters an aperture (i, j), reflected at $\left(X_{\mathrm{Mij}}-W+x_{\mathrm{Rij}}, Y_{\mathrm{Mij}}, \mathrm{z}_{\mathrm{RYij}}\right)$ by a mirror located at $y=Y_{\mathrm{Mij}}$, then reflected at $\left(X_{\mathrm{Mij}}, Y_{\mathrm{Mij}}-W+y_{\mathrm{Rij}}, \mathrm{z}_{\mathrm{RX}}\right)$ by a mirror located at $x=X_{\mathrm{Mij}}$, and exits the aperture. In the side view, the $z$-component of the image position is obtained as

$$
Z_{\mathrm{I}}=2 z_{\mathrm{RYij}}-Z_{\mathrm{S}}
$$

$2 z_{\mathrm{RY}} Z_{\mathrm{S}}$ because all lights that emitted from the same light source and that reflected at $z=z_{\mathrm{RY}}$ ij intersect the planar symmetrical position of the light source. The $x$-component $\left(x_{\mathrm{ZX}}\right)$ of the intersection of the extension of the reflected light and the line $z=2 z_{\mathrm{Rij}}-Z_{\mathrm{S}}$ is analyzed in order to confirm the image formation by the crossed-mirror array.

After some geometrical calculations, we obtain

$$
z_{\mathrm{RX} i \mathrm{ij}}=z_{\mathrm{RY} \mathrm{ij}}+\frac{\left(z_{\mathrm{RY} i \mathrm{j}}-Z_{\mathrm{S}}\right)\left(W-x_{\mathrm{RY} i \mathrm{j}}\right)}{X_{\mathrm{Mij}}-X_{\mathrm{S}}-W+x_{\mathrm{RY} i j}}
$$

and

$$
x_{\mathrm{ZX}}=X_{\mathrm{Mij}}-\frac{\left(X_{\mathrm{Mij}}-X_{\mathrm{S}}\right)\left(2 z_{\mathrm{RY} i j}-Z_{\mathrm{S}}-z_{\mathrm{RXij}}\right)}{z_{\mathrm{RX} \mathrm{ij}}-Z_{\mathrm{S}}} .
$$




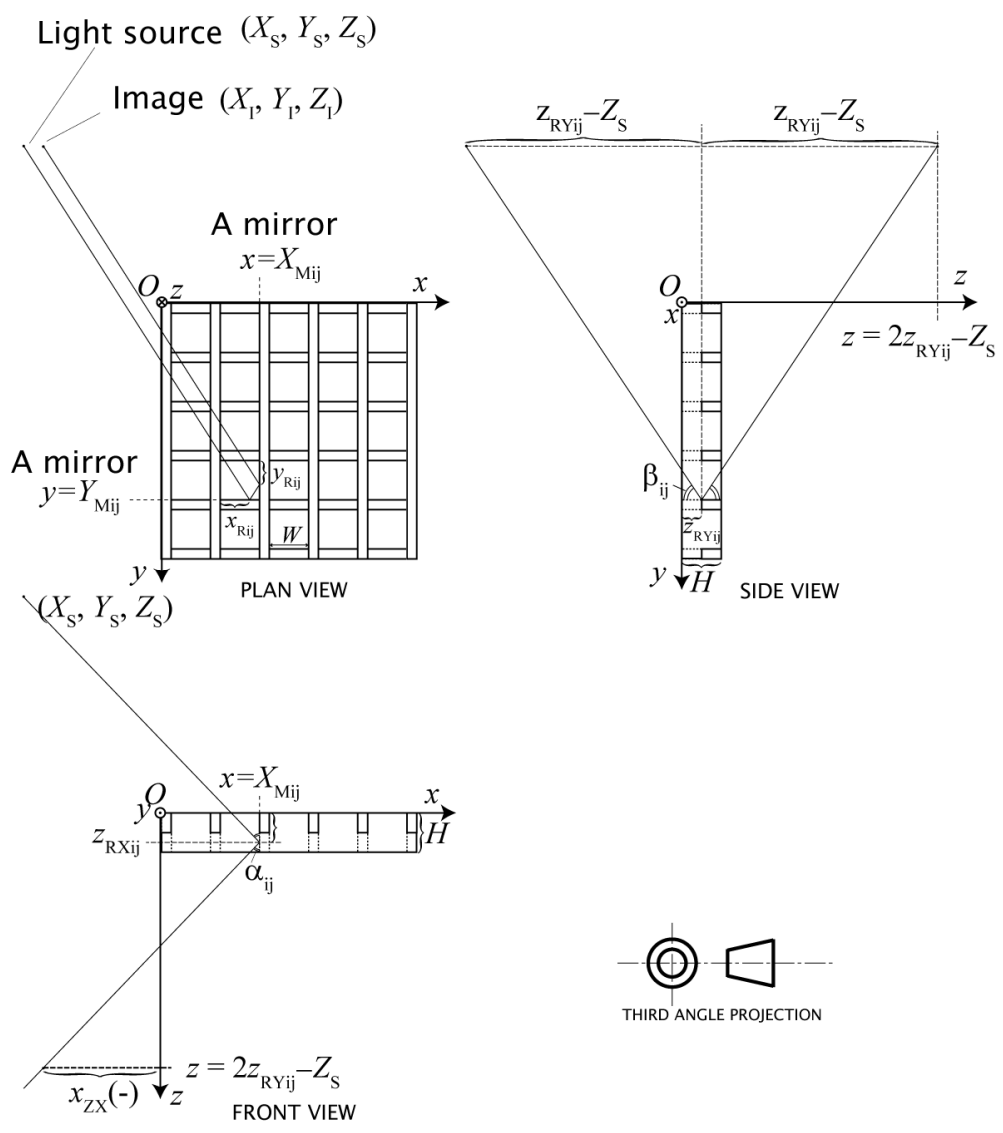

Figure 5. Coordinates and parameters to analyze the image formation by use of a crossed-mirror array. The origin of the coordinates is placed on the surface of the crossed-mirror array.

By substituting Eq. (2) into Eq. (3), the $x$-coordinate of the intersection of the exit light rays are expressed by

$$
x_{\mathrm{ZX}}=X_{\mathrm{S}}+2\left(W-x_{\mathrm{Rij}}\right) \text {. }
$$

Note that $x_{\mathrm{ZX}}$ is independent from the mirror position $X_{\mathrm{Mij}}$ and $Y_{\mathrm{Mij}}$. Therefore, all the incident lights into $x_{\mathrm{Rij}}$ position in each aperture are focused into the same position, which means

$$
X_{\mathrm{I}}=X_{\mathrm{S}}+2\left(W-x_{\mathrm{Rij}}\right) \text {. }
$$

Eq. (5) can be also derived by geometrical calculation in $x y$-plane (PLAN VIEW). By comparing the PLAN VIEW and SIDE VIEW, $y$-component of the image position is easily obtained as

$$
Y_{\mathrm{I}}=Y_{\mathrm{S}} \text {. }
$$

Consequently, light rays that firstly reflect at mirrors parallel to the $x$-axis at $x_{\mathrm{Rij}}$ from the left side converge to the image position that is shifted $2\left(W-x_{\mathrm{Rij}}\right)$ to the $x$ direction. Based on the symmetry of the configuration, light rays that firstly reflect at mirrors parallel to the $y$-axis at $y_{\text {Rij }}$ from the left side converge to the image position that is shifted $2\left(W-y_{\text {Rij }}\right)$ to the $y$ direction.

Because $0 \leq x_{\mathrm{Rij}} \leq W$ and $0 \leq y_{\mathrm{Rij}} \leq W$, the spread of the formed image of a point light source is $W$ in $x$ direction and $y$ direction. Thus, the point spread function can be enlarged by using a wide aperture of CMA. 
Current LED panels for digital signage have pitch of centimeter order. Therefore, appropriate size of the spread function is sub-centimeter. Another benefit in using large aperture CMA is expected to be a wide field angle, because wide aperture prevents higher reflections in the aperture. Thus, the proposed large aperture CMA is expected to satisfy wide field of angle (over 45 degrees), which is required to form floating image of a large LED array.

\subsection{Field of angle of CMA}

Allowable incident angle is determined by conditions in order to cause double reflection in an aperture. Fig. 6 shows boundaries of the allowable incident angle, that is a field of angle to form image. The boundaries are determined by the condition single reflection occurs in this condition. The figure illustrates boundaries when an object position is located left-side of an aperture and light rays incident at the left end of the aperture. In this case, the allowable incident angle is between $\alpha_{1}$ and $\alpha_{2}$, where

$$
\alpha_{1}=\tan ^{-1}(W / H)
$$

and

$$
\alpha_{2}=\tan ^{-1}(2 W / H)
$$

These boundaries also exist vertically so that double reflections in both directions occurs. Therefore, we have conducted experiments in slanting mirror configurations.

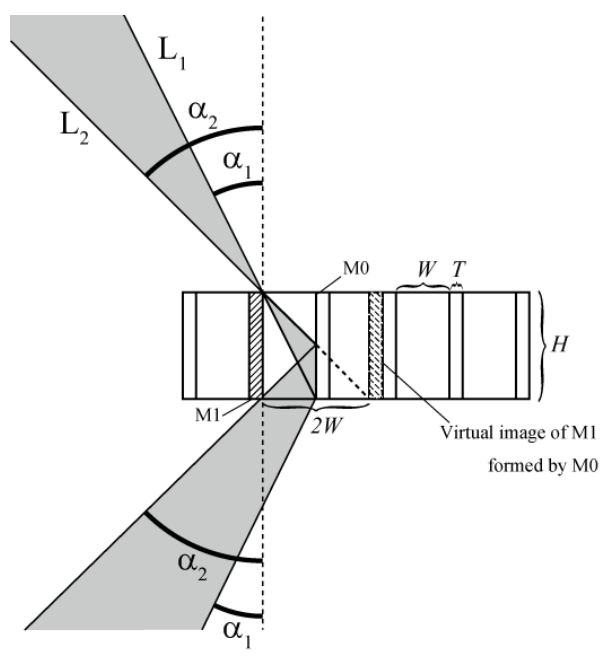

Figure 6. Schematic diagram showing the boundary of incident angles for an aperture. There are also boundaries for the reflection of the left-side mirror of the aperture. The other boundaries are bilateral symmetry of the lines.

\section{FLOATING LED DISPLAY BY USE OF CROSSED-MIRROR ARRAY}

\subsection{Focusing experiments by use of prototype CMA}

Requirements for optical components for public large digital signage include scalability, good rigidity, and safety under crashing. Thus, we decided to fabricate prototype CMA made of stainless mirrors. CMA was assembled from combshaped parts. Focusing of a point light source was investigated with the prototype CMAs. Experimental setups are shown in Fig. 7. A laser diode was used as the point light source. Reflected light distributions were investigated by changing the incident angle. Note that the plane-symmetric position of the source is located at 50-cm apart from the CMA. CMA with $4 \mathrm{~mm} \times 4 \mathrm{~mm}$ aperture and 8-mm height mirrors were used.

Fig. 8 shows observations of converging lights when incident angle $\theta$ was 15,30 , and 45 degrees. Screen distance was 10 $\mathrm{cm}, 30 \mathrm{~cm}$, and $50 \mathrm{~cm}$. In a close range to the CMA, double reflected light keeps grid-like shapes. At 30-cm distance, the light is converging but separations of bright spots are observed. At $50-\mathrm{cm}$ distance, that is, in the image plane, the bright spots converge together. 


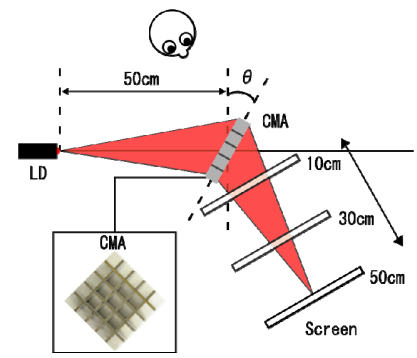

Figure 7. Experimental setup for observation of convergence of a focused laser diode light. The crossed-mirror array (CMA) was slanted in order to separate the double reflected lights from single reflected lights.

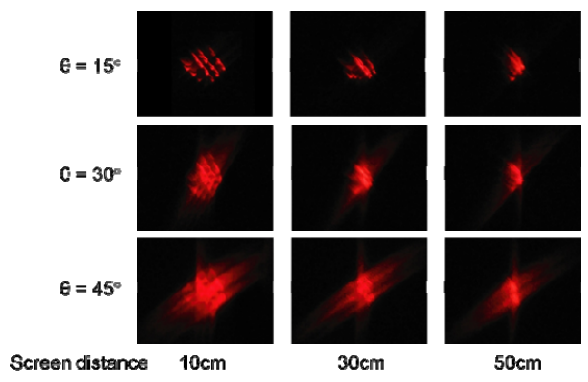

Figure 8. Observations of converging lights. Note that $50-\mathrm{cm}$ screen distance is geometrically derived image position. After double reflection, the lights from the nine apertures converge to the image position.

Light scattering was increased as the incident angle was increased to $45 \mathrm{deg}$. This is because 45 deg is the boundary condition expressed by Eq. (7) and causes light scatterings at the edges of the surface of CMA. However, the lights converged into the same position in the image plane. Convergence of the light was confirmed for all three incident angles, although reduction of scattering remains one of our future works.

\subsection{Floating LED sign by single CMA}

We have fabricated CMA for an LED array. Square shape apertures $(4 \mathrm{~mm} \times 4 \mathrm{~mm})$ are located diagonally, as shown in Fig. 9. There are $20 \times 20$ apertures. Fig. 10 and Video 1 show focusing of light to the floating position of LEDs. In close range and far range, LED lights were out of focus. At the focused distance, is clearly formed on the screen. Note that 'L' was flipped because the photographs were taken from the reverse side from the observer.

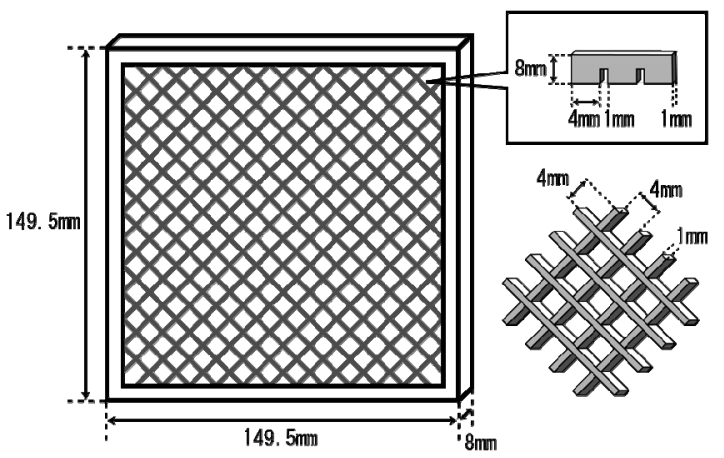

Figure 9. Diagram of a fabricated crossed-mirror array. Comb-shaped mirrors are combined to form the CMA.

Viewed images at different positions are shown in Fig. 11 and Video 2. In this experiment, a frame was placed at the image plane to indicate the position of the floating image. By moving the viewing position, motion parallax is occurred depending on the distance of the object from the viewing position. The floating ' $\mathrm{L}$ ' moves with the surrounding frame. Viewers perceived aerial image of ' $L$ ' is stable inside the frame. Thus, by using our fabricated CMA, floating image of an LED sign has been formed successfully. 

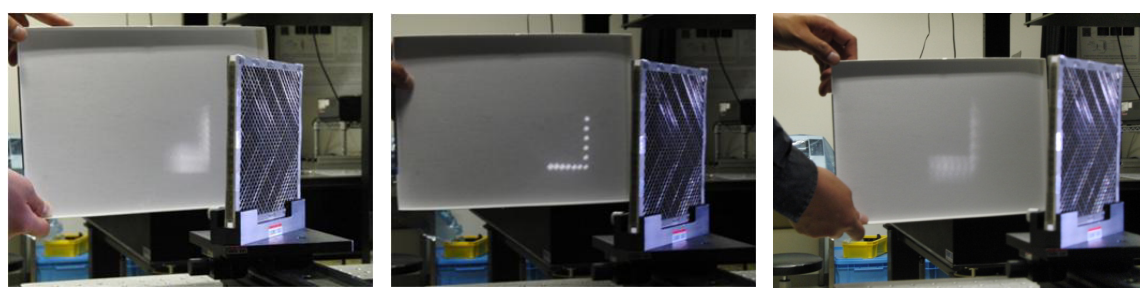

Figure 10. Aerial imaging of an LED array. The floating sign made of LED lamps are formed at the image position.

The image is significantly blurred when the screen is located at close ranges and long ranges.

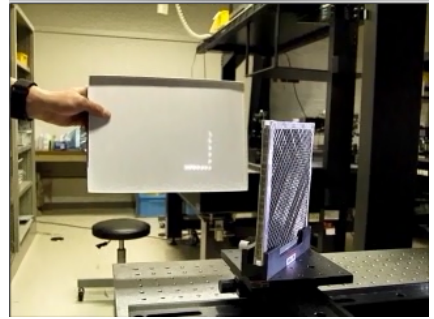

Video 1. Image formation and blurring by moving a screen: http://dx.doi.org/10.1117/12.909879.1
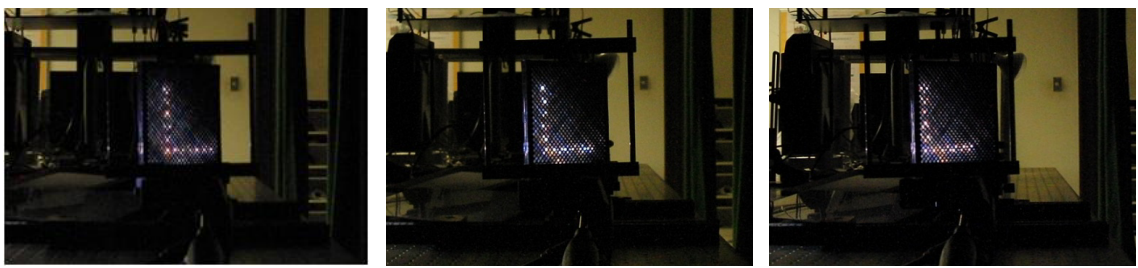

Figure 11. Observation of the floating LED sign at different viewing positions. A frame was located at the floating position. The floating letter ' $\mathrm{L}$ ' is stable inside the frame while changing the viewing positions.

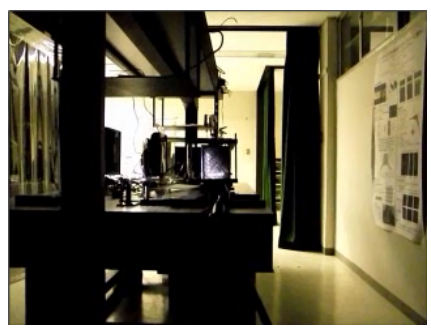

Video 2. Perceiving the floating sign by motion parallax. The bright ' $\mathrm{L}$ ' is a floating image inside a frame. The ' $\mathrm{L}$ ' is stable inside the frame while the video camera was moving: http://dx.doi.org/10.1117/12.909879.2

\subsection{Floating 3D LED signs by tiled CMAs}

We have fabricated three CMAs. The three CMAs are tiled side by side. LED signs of 'L', 'E', and 'D' are placed in different distance from the tiled CMAs. By changing the distance of the screen from the CMAs, one of the three LED signs was clearly formed while the others were blurred, as shown in Fig. 12 and Video 3. Thus, floating 3D LED signs have been successfully realized.
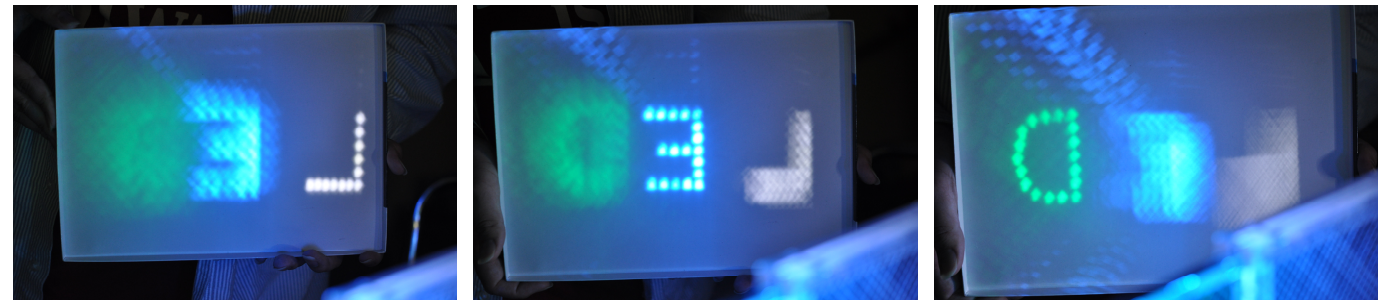

Figure 12. Formation of floating 3D LED signs. LED letters L, E, D are represented at different screen distances. 


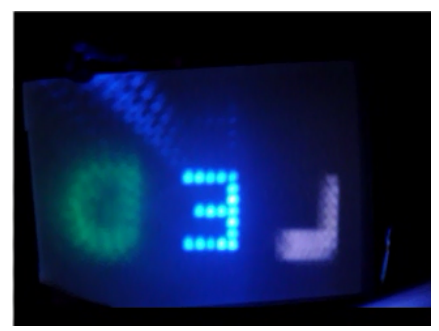

Video 3. Floating LED signs at three-dimensionally different positions: http://dx.doi.org/10.1117/12.909879.3

\section{CONCLUSION}

We have proposed a design of CMA for LED array. Analyses on image position of a point light source shows the point spread function of the CMA is as large as the aperture size. Light convergence at 45 deg was realized. In order provide a sub-centimeter point spread function and wide field angle, we have fabricated CMAs with 4-mm apertures and 8-mm mirror height. Floating display of LED signs in different depth was successfully realized by our fabricated CMAs.

\section{REFERENCES}

[1] Kaaret, P., Geissbühler, P., Chen, A., and Glavinas, E., "X-ray focusing using microchannel plages," Appl. Opt. 31 (34), 7339-7343 (1992).

[2] Chapman, H. N. and Rode, A. V., "Geometric optics of arrays of reflective surfaces," Appl. Opt. 33 (13), $2419-$ 2436 (1994).

[3] Peele, A. G., Nugent K. A., Rode, A. V., Gabel, K., Richardson, M. C., Strack, R., and Siegmund, W., "X-ray focusing with lobster-eye optics: a comparison of theory with experiment," Appl. Opt., 35 (22), 4420-4425 (1996).

[4] Gertsenshteyn, M., Jannson, T., and Savent, G., "Staring/focusing lobster-eye hard X-ray imaging for nonastronomical objects," Proc. SPIE 5922, 59220N (2005).

[5] Kawazu, M. and Inokuchi, T., "Imaging device using a roof mirror lens array," Appl. Opt., 24 (24), $4300-4306$ (1985).

[6] Maekawa, S., Nitta, K., and Matoba, O., "Transmissive optical imaging device with mirrormirror array," Proc. SPIE 6392, 63920E (2006).

[7] Miyazaki, D., Hirano, N., Maeda, Y., Ohno, K., and Maekawa, S., "Volumetric display using a roof mirror grid array," Proc. SPIE 7524, 75240N (2010).

[8] Yamamoto, H., Kouno, M., Muguruma, S., Hayasaki, Y., Nagai, Y., Shimizu, Y., and Nishida, N., "Enlargement of viewing area of stereoscopic full-color LED display by use of a parallax barrier," Appl. Opt., 41 (32), 6907-6919 (2002).

[9] Nishimura, H., Abe, T., Uchida, K., Yamamoto, H., Hayasaki, Y., and Nishida, N., "Development of 140-inch autostereoscopic display by use of full-color LED panel," Proc. SPIE 6486, 64861B (2007).

[10] Yamamoto, H., Nishimura, H., Uchida, K., Ono, K., Hayasaki, Y, and Suyama, S,. "Depth Perception for Moving Pictures Shown on a Large LED Display with an Aperture Grille," J. Soc. Inf. Display, 17 (12), 1031 1036 (2009).

[11] Yamamoto, H., Kimura, T., Matsumoto, S., and Suyama, S., "Viewing-Zone Control of Light-Emitting Diode Panel for Stereoscopic Display and Multiple Viewing Distances," J. Display Tech., 6 (9), 359-366 (2010).

[12] Yamamoto, H., Tsutsumi, M., Yamamoto, R., Kajimoto, K., and Suyama, S., "Development of high-frame-rate LED panel and its applications for stereoscopic 3D display," Proc. SPIE 7956, 79560R (2011). 